\section{Ein Auge riskiert}

Ein dreijähriger Junge wurde wegen einer massiv erweiterten rechten $\mathrm{Pu}$ pille in die Nothilfe gebracht (Abb. A). Die Eltern hatten die Veränderung an dem Kind festgestellt, nachdem es im Garten gespielt hatte. Eine halbe Stunde zuvor bemerkten die Eltern, dass der Junge geweint hatte. Die rechte Pupille reagierte nicht auf Licht und Akkommodation, ansonsten war die körperliche Untersuchung unauffällig.

Nach intensivem Befragen stellte sich heraus, dass der Junge die Blüte einer Engelstrompete (Abb. B) im Garten abgezupft und danach sich mit dem rechten Zeigefinger das rechte Auge gerieben hatte. Die Engelstrompete aus der Gattung Brugmansia ist eine Zierblume aus Südamerika, die aber
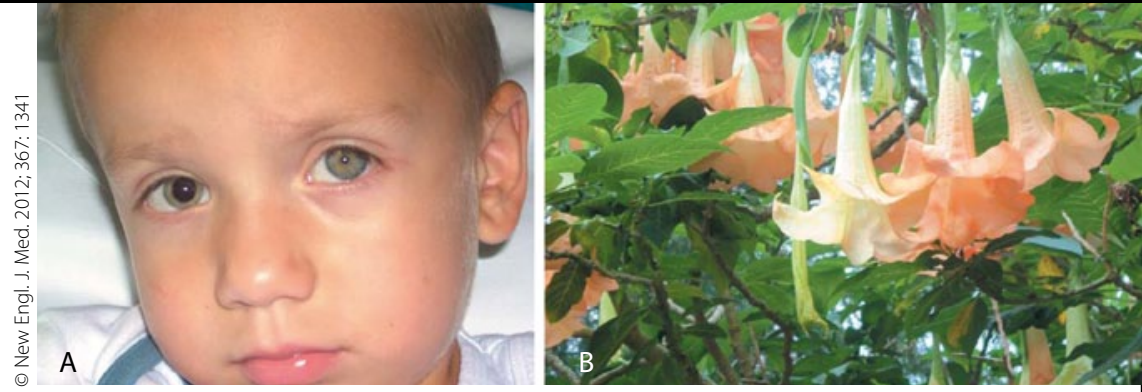

Einseitige Mydriasis bei einem Dreijährigen (A). Engelstrompete (B).

mittlerweile fast weltweit vorkommt und parasympathisch wirksame Alkaloide wie Scopolamin, Hyoscin und Atropin enthält. Stellt man bei einem gesunden Kind plötzlich eine einseitige Mydriasis ohne Lichtreaktion fest, so sollte als erstes an eine Kontamination mit Engelstrompete gedacht werden. Schwere Vergiftungen können zu Halluzinationen, Hyperthermie, Krampfanfällen, Lähmungen und Todesfällen führen. Im vorliegenden Fall lagen sonst keine toxischen Symptome vor, sodass die Eltern beraten und das Kind entlassen wurde. Die Mydriasis verschwand innerhalb von drei Tagen spontan.

\section{H. S. FÜESSL =}

\section{- A. Vunda und G. Alcoba}

(Korres.: University Hospitals of Geneva, Switzerland): Mydriasis in the garden. New Engl. J. Med. 2012; 367: 1341

\title{
HES-Akten geschlossen
}

Die seit Jahrzehnten geführte Diskussion, ob Hydroxyethylstärke (HES) gegenüber Kochsalzlösung für Intensivpatienten Vorteile habe, scheint nun durch eine große Publikation beendet.

— Hydroxyethylstärke (HES) nimmt im Kapitel der Wissenschaftsfälschungen eine besondere Rolle ein. Ende 2010 wurde ein deutscher anästhesiologischer Chefarzt mit sofortiger Wirkung seines Amtes enthoben, weil er in 89 Fachartikeln günstige Daten über HES frei erfunden hatte. Die gute Meinung über HES beruhte also auf Fälschungen.

Nun wurde in einer randomisierten Studie an 7000 Patienten von Intensivstationen in der Krankenhäusern von Australien und Neuseeland gezeigt, dass 6\%-ige HES mit einem Molekulargewicht von $130 \mathrm{kD}$ gegenüber $0,9 \%$-iger Kochsalzlösung als Volumenersatz nicht nur keine Vorteile, sondern möglicherweise sogar Nachteile hat. Im Verlauf von 90 Tagen verstarben von 3315 Pati-

enten der HES-Gruppe 597 (18\%), von 3336 der Kochsalzgruppe 566 (17\%). Auch bei differenzierter Betrachtung der Mortalität in sechs prädefinierten Untergruppen ergaben sich keine Unterschiede.

7\% der Patienten unter HES und $5,8 \%$ unter Kochsalz mussten sich einer Nierenersatztherapie unterziehen. Dieser Unterschied war statistisch eben signifikant. Unerwünschte Nebenwirkungen waren mit $5,3 \%$ vs. $2,8 \%$ hochsi-

\section{Kommentar}

Man kann einwenden, dass die Mortalität der Intensivpatienten in der Studie niedriger war als erwartet. Patienten mit intrakraniellen Blutungen und solche, die nach Einschätzung des aufnehmenden Arztes nicht überleben würden, wurden aus der Studie ausgeschlossen. Insofern mag die untersuchte Patientenpopulation nicht repräsentativ für alle schwerkranken Patienten von Intensivstationen und vor allem schwer verletzte Unfallopfer sein. Daher bleibt ein gnifikant häufiger unter HES. Insbesondere beobachtete man Hauterscheinungen wie Juckreiz und Exantheme, was wahrscheinlich auf die Einlagerung der Mikropartikel in das retikuloendotheliale System zurückzuführen ist. Auf diese Weise kann es auch zu akuten Schäden an Nieren und Leber kommen.

\section{- A. Myburgh et al.}

Hydroxyethyl starch or saline for fluid resuscitation in intensive care. New Engl. J. Med. 2012; online; DOI: 10.1056/NEJMoal1209759

\section{kleines Fragezeichen, ob HES bei solchen} Patienten in der Erstversorgung am Unfallort nicht doch Vorteile besitzt. Wenn sie denn vorhanden sind, so werden sie allerdings durch klare Nachteile hinsichtlich möglicher Nierenschäden erkauft. Keine guten Jahre für HES: 2010 wurden die positiven Daten als Fälschung aufgedeckt, 2012 in einer seriösen Studie negative Daten publiziert.

H. S. FÜESSL 